\title{
Sender- and Receiver-centered Interference in Wireless Ad Hoc Networks
}

\author{
Lucia Gallina and Sabina Rossi \\ Dipartimento di Informatica, Università Ca' Foscari \\ Venezia, Italy \\ E-mail: $\{$ lgallina,srossi $\} @$ dsi.unive.it
}

\begin{abstract}
Energy consumption in general and interference in particular are among the most critical issues in wireless networks. In this paper we present the E-BUM calculus, a Energy-aware calculus for Broadcast, Unicast and Multicast communications in wireless ad hoc networks. We formalize the notions of senderand receiver-centered interference and provide efficient proof techniques for verifying the absence of interference between a specific set of nodes.
\end{abstract}

Keywords_process algebra; manets; interference; topology control

\section{INTRODUCTION}

Wireless ad-hoc networks consist of autonomous mobile devices communicating with each other via radio transceivers through the protocol IEEE 802.11 (WiFi). This type of communication has a physical scope, because a radio transmission spans over a limited area. Moreover, nodes are primarily powered by a weak battery and thus energy consumption is among the foremost critical issues for network lifetime. The main goal of topology control is to reduce node power consumption in order to extend the lifetime of the network. This can be considered a trade-off between power saving and network connectivity: choosing a low transmission power for a node will reduce its power consumption, but it will also possibly reduce its connectivity with the other nodes in the network. One of the main approaches to reducing energy consumption consists in minimizing interference between the network nodes. In the context of topology control, interference is usually confined by constructing sparse topologies or topologies with low node degrees, without providing rigorous motivations or proofs.

The main contribution of this paper is the definition of a energy-aware model for wireless ad-hoc networks as well as the formalization of two different notions of interference.

We first present the E-BUM calculus, a calculus for Energyaware Broadcast, Unicast and Multicast communications in wireless ad-hoc networks [5]. It allows us to model the ability of a node to broadcast a message to any other node within its physical transmission range, and to move in and out of the transmission range of other nodes in the network. The connectivity of a node is represented by a location and a transmission radius. Broadcast communications are limited to the transmission cell of the sender, while unicast and multicast communications are modelled by specifying, for each output action, the addresses of the intended recipients of the message. Moreover, arbitrary and unexpected connections and disconnections of nodes as well as the possibility for a node to dynamically adjust its transmission power are modelled by enabling nodes to modify the corresponding transmission radius.

Based on the E-BUM model, we formally introduce two different definitions of interference: a sender-centered definition which measures the number of nodes potentially disturbed by the sender of a message, and a receiver-centered definition which gives a measure of the number of nodes potentially disturbing a given receiver. These two definitions are based on the notion of observability that pertains to the semantics of our calculus: what we observe of a transmission is its ability to reach the set of its intended receivers. Efficient proof techniques for verifying the absence of interference between a specific set of nodes are also proposed.

\section{RELATED WORK}

Many researchers have proposed algebraic models for wireless ad-hoc networks. The E-BUM calculus presented in this paper is an extension of CMN (Calculus of Mobile Ad-Hoc Networks) [7] that models unicast and multicast communications as well as the ability for a node to control its transmission power. Related to our model is also the $\omega$-calculus [11], a conservative extension of the $\pi$-calculus, which deals with unicast and multicast communications by allowing two nodes to share a private channel (hidden to the external network). We believe that our model better represents the real nature of wireless ad-hoc networks: a message sent to a specific group of receivers is not hidden to the rest of the network and all the nodes within the transmission cell of the sender will be able to receive the message anyway.

As mentioned above, reducing interference is one of the main goals of topology control besides direct energy conservation by restriction of transmission power. Most of the proposed topology control algorithms try to reduce interference implicitly as a consequence of sparseness or low degree of the resulting topology graph. An explicit concept of interference, based on the current network traffic, has been proposed in [8], while an explicit definition that is independent on the network traffic has been presented in [1]. This definition is based on the question how many nodes are affected by communication over a given link. In contrast the definition 
presented in [4] considers interference at the intended receiver of a message. We are not aware of any work addressing the problem of verifying the absence of a certain degree of interference between a specific set of nodes.

\section{THE CALCULUS}

We introduce the E-BUM calculus that models a wireless adhoc network as a collection of nodes, running in parallel, and using channels to broadcast messages. This calculus extends CMN [7] to support multicast and unicast communications. Moreover, it allows one to model the arbitrary and unexpected connections and disconnections of nodes in a network as well as the possibility for a node to administrate energy consumption by choosing the optimal transmission radius to communicate with the desired receivers.

Syntax: We use letters $c$ and $d$ for channels; $m$ and $n$ for nodes; $l, k$ and $h$ for locations; $r$ for transmission radii; $x$, $y$ and $z$ for variables. Closed values contain nodes, locations, transmission radii and any basic value (booleans, integers, ...). Values include also variables. We use $u$ and $v$ for closed values and $w$ for (open) values. We denote by $\tilde{v}, \tilde{w}$ tuples of values.

The syntax of E-BUM is shown in Table I. This is defined in a two-level structure: the lower one for processes, the upper one for networks. Networks are collections of nodes (which represent devices), running in parallel, using channels to communicate messages. As usual, $\mathbf{0}$ denotes the empty network and $M_{1} \mid M_{2}$ represents the parallel composition of two networks. Processes are sequential and live within the nodes. Process $\mathbf{0}$ denotes the inactive process. Process $c(\tilde{x}) . P$ can receive a tuple $\tilde{w}$ of (closed) values via channel $c$ and continue as $P\{\tilde{w} / \tilde{x}\}$, i.e., as $P$ with $\tilde{w}$ substituted for $\tilde{x}$ (where $|\tilde{x}|=|\tilde{w}|)$. Process $\bar{c}_{L, r}\langle\tilde{w}\rangle \cdot P$ can send a tuple of (closed) values $\tilde{w}$ via channel $c$ and continue as $P$. The tag $L$ is used to maintain the set of locations of the intended recipients: $L=\infty$ represents a broadcast transmission, while a finite set of locations $L$ denotes a multicast communication (unicast if $L$ is a singleton). The tag $r$ represents the power of the transmission: we assume that the choice of the transmission power may depend on precise strategies which are implemented in the communication protocol; hence it is reasonable considering the transmission radius of a communication as an information given by the process running in the sender node. Notice that a node $n$ will never execute any output action requiring a transmission radius $r$ with $r>r_{n}$. Syntactically, the tags $L$ and $r$ associated to the channel $c$ in an output action may be variables, but they must be instantiated when the output prefix is ready to fire. Process $\left[w_{1}=w_{2}\right] P, Q$ behaves as $P$ if $w_{1}=w_{2}$, and as $Q$ otherwise. We write $A\langle\tilde{w}\rangle$ to denote a process defined via a (possibly recursive) definition $A(\tilde{x}) \stackrel{\text { def }}{=} P$, with $|\tilde{x}|=|\tilde{w}|$, where $\tilde{x}$ contains all channels and variables that appear free in $P$.

We write $n[P]_{l, r}^{\mu}$ for a node named $n$ (this is the logic location of the device in the network), located at $l$, with transmission radius $r$, mobility tag $\mu$, and executing the process $P$. The tag $\mu$ is $\mathrm{m}$ for mobile nodes, and $\mathrm{s}$ for stationary nodes; $l$ denotes the physical location of the node.
Nodes cannot be created or destroyed. To each node $n$ is also associated a maximum transmission radius $r_{n}$; nodes may control power consumption by dynamically adjusting their transmission radius $r$ provided that $r \in\left\{0, \ldots, r_{n}\right\}$ (note that the transmission radius of a communication is straightly proportional to the power). Clearly, if $r=0$ then the node is disconnected. It is reasonable to assume that the radius varies within a finite set of discrete values, corresponding to the range of frequencies used to communicate. The possibility that nodes communicate with each other is verified looking at the physical locations and the transmission radius of the sender, in other words if a node broadcasts a message, this information will be received only by the nodes that lie in the area delimited by the transmission radius of the sender.

In the process $c(\tilde{x}) . P$, the tuple $\tilde{x}$ is bound in $P$. We denote by $\prod_{i \in I} M_{i}$ the parallel composition of networks $M_{i}$, for $i \in I$. We write $c_{l}$ for $c_{\{l\}}, \bar{c}_{L}\langle w\rangle$ for $\bar{c}_{L}\langle w\rangle . \mathbf{0}, \mathbf{0}$ for $n[\mathbf{0}]_{l, r}^{\mu}$ and $\left[w_{1}=w_{2}\right] P$ for $\left[w_{1}=w_{2}\right] P, \mathbf{0}$. We assume that there are no free variables in a network (while there can be free channels). Moreover, we assume that in any network each node identifier is unique and the corresponding transmission radius is compatible with the node power capacity. Formally, a network $M \equiv n_{1}\left[P_{1}\right]_{l_{1}, r_{1}}^{\mu_{1}}\left|n_{2}\left[P_{2}\right]_{l_{2}, r_{2}}^{\mu_{2}} \ldots\right| n_{k}\left[P_{k}\right]_{l_{k}, r_{k}}^{\mu_{k}}$ is wellformed if for $i, j \in\{1, \ldots, k\}$ it holds that $n_{i} \neq n_{j}$ when $i \neq j$ and $r_{i} \in\left\{0, \ldots, r_{n_{i}}\right\}$.

Reduction Semantics: The dynamics of the calculus is specified by the reduction relation $(\rightarrow)$ over networks, described in Table II. As usual, it relies on an auxiliary relation, called structural congruence $(\equiv)$, such that for instance $M|N \equiv N| M,(M \mid N)\left|M^{\prime} \equiv M\right|\left(N \mid M^{\prime}\right)$ and $M \mid \mathbf{0} \equiv M$ (see [5] for full details). We assume the possibility of comparing locations in order to determine whether a node lies or not within the transmission cell of another node. This is done through the function $d(\cdot, \cdot)$ which takes two locations and returns their distance.

Rule (R-Bcast) models the transmission of a tuple $\tilde{v}$ through a channel $c_{L, r}$. The set $L$ associated to channel $c$ indicates the locations of the intended recipients. Indeed, nodes communicate using radio frequencies that enable only broadcast messages (monopolizing channels is not permitted). However, a node may decide to communicate with a specific node (or group of nodes), this is the reason why we decided to associate to each output action a set of transmission recipients. The cardinality of this set indicates the kind of communication: if $L=\infty$ then the recipients set is the whole network and a broadcast transmission is performed, while if $L$ is a finite set (resp., a singleton) then a multicast (resp., a unicast) communication is realized. A radius $r$ is also associated to the channel $c$, indicating the transmission radius required for that communication which may depend on the power consumption strategy adopted by the surrounding protocol.

In our calculus transmission is a non-blocking action: transmission proceeds even if there are no nodes listening for messages. The messages transmitted will be received only by those nodes which lie in the transmission area of the sender. It may occur that some receivers within the range of the 


\begin{tabular}{clll}
\hline Networks & & Processes \\
$\mathrm{M}, \mathrm{N}::=\mathbf{0}$ & Empty network & $\mathrm{P}, \mathrm{Q}, \mathrm{R}::=\mathbf{0}$ & \\
$\left|M_{1}\right| M_{2}$ & Parallel composition & $\mid c(\tilde{x}) . P$ & Inactive process \\
$\mid n[P]_{l, r}^{\mu}$ & Node (or device) & $\mid \bar{c}_{L, r}\langle\tilde{w}\rangle . P$ & Input \\
& & $\mid\left[w_{1}=w_{2}\right] P, Q$ & Output \\
& & $\mid A\langle\tilde{w}\rangle$ & Matching \\
& & Recursion \\
\hline
\end{tabular}

TABLE I: Syntax

transmitter do not receive the message. This may be due to several reasons that concern the instability and dynamism of the network. In terms of observation this corresponds to a local activity of the network which an observer is not party to.

In our calculus we consider connections, disconnections and movements of nodes. All these actions are atomic, e.g., while moving, a node cannot do anything else. Rule (R-Rad) models the possibility for a node $n$ to control power consumption by changing its transmission radius $r$ into $r^{\prime}$ provided that $r^{\prime} \in$ $\left\{0, \ldots, r_{n}\right\}$. Notice that a node is disconnected when its radius is set to 0 . Rule (R-Move) models arbitrary and unpredictable movements of mobile nodes. $\delta$ denotes the maximum distance that a node can cover in a computational step. The remaining rules are standard in process calculi. We denote by $\longrightarrow^{*}$ the reflexive and transitive closure of $\longrightarrow$.

Behavioral Semantics: The central actions of our calculus are transmission and reception of messages. However, only the transmission of messages (over unrestricted channels) can be observed. An observer cannot be sure whether a recipient actually receives a given value. Instead, if a node receives a message, then surely someone must have sent it. Following [9], we use the term barb as a synonymous of observable. In our definition of barb a transmission is observable only if at least one location in the set of the intended recipients is able to receive the message.

Definition 3.1: [Barb] We write $M \downarrow_{c}$ if $M$ is of the form $n\left[\bar{c}_{L, r}\langle\tilde{v}\rangle . P\right]_{l, r}^{\mu} \mid M^{\prime}$ and $\exists k \in L \wedge d(l, k) \leqslant r$. We write $M \Downarrow_{c}$ if $M \longrightarrow^{*} M^{\prime} \downarrow_{c}$.

Notice that, if $M \equiv n\left[\bar{c}_{L, r}\langle\tilde{v}\rangle . P\right]_{l, r}^{\mu} \mid M^{\prime}$ and $M \downarrow_{c}$ then at least one of the recipients in $L$ is actually able to receive the message.

To define our observation equivalence we will ask for the largest relation which satisfies the following properties. Let $\mathcal{R}$ be a relation over networks:

Barb preservation. $\mathcal{R}$ is barb preserving if $M \mathcal{R} N$ and $M \downarrow_{c}$ implies $N \Downarrow_{c}$.

Reduction closure. $\mathcal{R}$ is reduction closed if $M \mathcal{R} N$ and $M \longrightarrow M^{\prime}$ implies that there exists $N^{\prime}$ such that $N \longrightarrow{ }^{*} N^{\prime}$ and $M^{\prime} \mathcal{R} N^{\prime}$.

Contextuality. $\mathcal{R}$ is contextual if $M \mathcal{R} N$ implies $\mathcal{C}[M] \mathcal{R} \mathcal{C}[N]$ for any context $\mathcal{C}[\cdot]$, where a context is a network term with a hole [.] defined by:

$$
\mathcal{C}[\cdot]::=[\cdot]|[\cdot]| M|M|[\cdot] .
$$

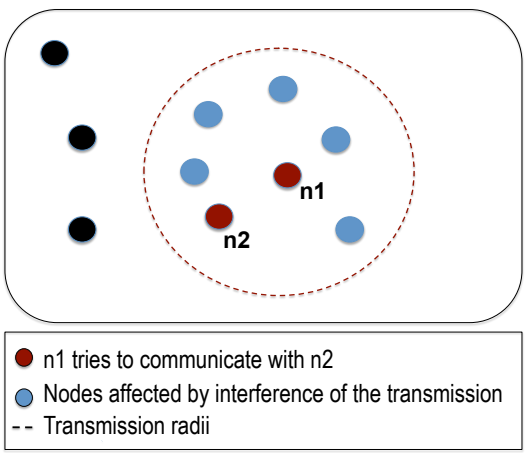

Fig. 1: Example of interference caused by a transmission

Definition 3.2: [Reduction barbed congruence] Reduction barbed congruence, written $\cong$, is the largest symmetric relation over networks, which is reduction closed, barb preserving, and contextual.

Two networks are related by $\cong$ if they exhibit the same behaviour (communications) relative to the corresponding sets of intended receivers. In [5] a bisimulation-based proof technique for $\cong$ is developed. It provides an efficient method to check whether two networks are related by $\cong$.

\section{SENDER-CENTERED INTERFERENCE}

Following the definition introduced in [1], the notion of sender-centered interference arises from a natural question: How many nodes are disturbed by a given communication over the network?

Consider the situation depicted in Figure 1 where a node $n 1$ is intended to transmit a message to $n 2$. We can define sender-centered interference as the number of nodes listening to the message, but not interested in receiving it.

Definition 4.1: [Level of Sender-centered Interference] Let $c_{L} ! \tilde{v}[l, r]$ be an output action, $H$ be the set of possible locations of the nodes in the network and $K=\{k \in H$ : $d(l, k) \leq r\}$. The level of sender-centered interference relative to this output is defined as:

$$
I_{\text {send }}\left(c_{L} ! \tilde{v}[l, r]\right)=|K-L| .
$$

If the set of nodes not interested in receiving the message is empty, i.e., $I_{\text {send }}\left(c_{L} ! \tilde{v}[l, r]\right)=0$, then we can affirm that the transmission $c_{L} ! \tilde{v}[l, r]$ does not provoke any interference. 


$$
\begin{array}{cc}
\text { (R-Bcast) } \frac{r \neq 0, \forall i \in I \cdot d\left(l, l_{i}\right) \leq r, r_{i} \neq 0,\left|\tilde{x}_{i}\right|=|\tilde{v}|}{n\left[\bar{c}_{L, r}\langle\tilde{v}\rangle \cdot P\right]_{l, r}^{\mu}\left|\prod_{i \in I} n_{i}\left[c\left(\tilde{x}_{i}\right) \cdot P_{i}\right]_{l_{i}, r_{i}}^{\mu_{i}} \rightarrow n[P]_{l, r}^{\mu}\right| \prod_{i \in I} n_{i}\left[P_{i}\left\{\tilde{v} / \tilde{x}_{i}\right\}\right]_{l_{i}, r_{i}}^{\mu_{i}}} \\
\begin{array}{cc}
\text { (R-Rad) } \frac{r^{\prime} \in\left\{0, \ldots, r_{n}\right\}}{n[P]_{l, r}^{\mu} \rightarrow n[P]_{l, r^{\prime}}^{\mu}} & \text { (R-Move) } \frac{d(l, k) \leq \delta}{n[P]_{l, r}^{\mathrm{m}} \rightarrow n[P]_{k, r}^{\mathrm{m}}} \\
\text { (R-Par) } \frac{M \rightarrow M^{\prime}}{M\left|N \rightarrow M^{\prime}\right| N} & \text { (R-Struct) } \frac{M \equiv N N \rightarrow N^{\prime} N^{\prime} \equiv M^{\prime}}{M \rightarrow M^{\prime}}
\end{array}
\end{array}
$$

TABLE II: Reduction Semantics

The E-BUM calculus allows us to observe the case in which a transmission reaches only its intended receivers, without any interference. Indeed, we can compare the behaviour of a node communicating with a given set $L$ of recipients, with the behaviour of the same node but broadcasting all its communications to the whole network. If the two behaviours are related by $\cong$, then we can affirm that the node transmissions do not provoke any interference, in other words they do not disturb any other node in the network.

Let us first define the broadcasting version of a process $P$, denoted by $\operatorname{brd}(P)$, as follows:

- if $P=\mathbf{0}$ then $\operatorname{brd}(P)=\mathbf{0}$

- if $P=c(\tilde{x}) \cdot P^{\prime}$ then $\operatorname{brd}(P)=c(\tilde{x}) \cdot \operatorname{brd}\left(P^{\prime}\right)$

- if $P=\bar{c}_{L, r}\langle\tilde{v}\rangle \cdot P^{\prime}$ then $\operatorname{brd}(P)=\bar{c}_{\infty, r}\langle\tilde{v}\rangle \cdot \operatorname{brd}\left(P^{\prime}\right)$

- if $P=\left[w_{1}=w_{2}\right] Q, R$ then $\operatorname{brd}(P)=\left[w_{1}=w_{2}\right] \operatorname{brd}(Q), \operatorname{brd}(R)$.

We provide an efficient proof technique for verifying the absence of sender-centered interference for a specific node $n$.

Definition 4.2: [Absence of sender-centered Interference] We say that a node $n[P]_{l, r}^{\mu}$ is free of sender-centered interference if $n[P]_{l, r}^{\mu} \cong n[b r d(P)]_{l, r}^{\mu}$.

Notice that, by contextuality, if $n[P]_{l, r}^{\mu} \cong n[\operatorname{brd}(P)]_{l, r}^{\mu}$ then also $n[P]_{l, r}^{\mu}\left|M \cong n[\operatorname{brd}(P)]_{l, r}^{\mu}\right| M$ for any network $M$. This means that if $n[P]_{l, r}^{\mu}$ is free of sender-centered interference, then it so independently of the behaviour of the other nodes in the network.

The following theorem proves the soundness of the above technique. The proof is reported in [5].

Theorem 4.3: If $n[P]_{l, r}^{\mu}$ is free of sender-centered interference then for all output actions $c_{L} ! \tilde{v}[l, r]$ performed by $n[P]_{l, r}^{\mu}$ it holds that

$$
I_{\text {send }}\left(c_{L} ! \tilde{v}[l, r]\right)=0 .
$$

We may be interested in verifying the absence of sendercentered interference relative to a specific set of nodes $S$. This can be done by defining the broadcasting version of a process $P$ relative to $S$, noted $\operatorname{brd}(S, P)$. The definition of $\operatorname{brd}(S, P)$ analogous to the one of $\operatorname{brd}(P)$ except for the third item that is

- if $P=\bar{c}_{L, r}\langle\tilde{v}\rangle \cdot P^{\prime}$ then

$$
\operatorname{brd}(P, S)=\bar{c}_{L \cup S, r}\langle\tilde{v}\rangle . \operatorname{brd}\left(P^{\prime}, S\right)
$$

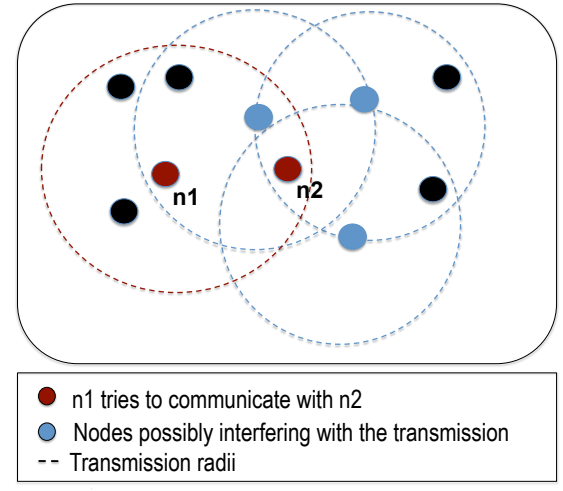

Fig. 2: Example of interference suffered by a transmission

In this case, we obtain that a node $n[P]_{l, r}^{\mu}$ is free of sendercentered interference relative to $S$ if $n[P]_{l, r}^{\mu} \cong n[\operatorname{brd}(P, S)]_{l, r}^{\mu}$.

\section{RECEIVER-CENTERED INTERFERENCE}

We now formalize the notion of interference at the intended receiver of a message (see [12], [4]). Consider the situation depicted in Figure 2: $n 1$ is trying to transmit a message to $n 2$, but $n 2$ lies in the transmission cell of three other devices, which, due to their transmissions, may prevent $n 2$ to receive the message sent by $n 1$.

Before giving a definition of receiver-oriented interference, we need to make an assumption about the set of possible recipients of the devices interacting in a network. We assume that for each process $P$ executed by a network node, it is possible to identify the set of all the intended recipients that may appear in an output action performed by $P$. We denote by $\operatorname{dest}(P)$ the minimum set of locations ensuring that for each output action $\bar{c}_{L, r}\langle w\rangle$ performed by $P$ it holds that $L \in \operatorname{dest}(P)$. Following we introduce the level of receivercentered interference as an upper bound of the quantity of noise possibly provoked by a network $M$ to a location $l$.

Definition 5.1: [Level of Receiver-centered Interference] Let $M$ be a network consisting of $k$ devices:

$$
M=n_{1}\left[P_{1}\right]_{l_{1}, r_{1}}^{\mu_{1}}|\ldots| n_{k}\left[P_{k}\right]_{l_{k}, r_{k}}^{\mu_{k}},
$$

then the level of receiver-centered interference with respect to a given location $l$ is defined as: 


$$
\begin{gathered}
I_{r e c}(l, M)=\mid\left\{j \in\{1, \ldots, k\} . d\left(l, l_{j}\right) \leq r_{n_{j}} \wedge\right. \\
\left.l \notin \operatorname{dest}\left(P_{j}\right) \wedge \operatorname{dest}\left(P_{j}\right) \neq 0\right\} \mid
\end{gathered}
$$

where $r_{n_{j}}$ indicates the maximum transmission radius the node $n_{j}$ may choose for its communications. The last condition, $\operatorname{dest}\left(P_{j}\right) \neq 0$, ensures that only those nodes which are active, i.e., execute at least one output action, are considered.

As we have done above, we use the E-BUM calculus to provide an efficient proof technique for the ideal situation where a location $l$ is reached only by those nodes which are interested in communicating with it.

Let us write $\operatorname{brd}(P, l)$ for $\operatorname{brd}(P,\{l\})$ (see definition above). Moreover, given a network $M$ consisting of $k$ devices:

$$
M=n_{1}\left[P_{1}\right]_{l_{1}, r_{1}}^{\mu_{1}}|\ldots| n_{k}\left[P_{k}\right]_{l_{k}, r_{k}}^{\mu_{k}}
$$

we write $\operatorname{brd}(M, l)$ for

$$
n_{1}\left[\operatorname{brd}\left(P_{1}, l\right)\right]_{l_{1}, r_{1}}^{\mu_{1}}|\ldots| n_{k}\left[\operatorname{brd}\left(P_{k}, l\right)\right]_{l_{k}, r_{k}}^{\mu_{k}} .
$$

Definition 5.2: [Absence of receiver-centered Interference] We say that a location $l$ is free of receiver-centered interference with respect to a network $M$ if,

$$
M \cong \operatorname{brd}(M, l) \text {. }
$$

Notice that, by contextuality, if $l$ is free of receiver-centered interference with respect to $M$ then for any node $n$ placed at location $l$, and for any radius $r$, mobility tag $\mu$ and process $P$, we have

$$
n[P]_{l, r}^{\mu}\left|M \cong n[P]_{l, r}^{\mu}\right| \operatorname{brd}(M, l) .
$$

If we are interested in proving the absence of receivercentered interference for a location $l$ in a network $M$, but relative to a specific set of nodes $S$, then we can proceed as follows. First, we split $M$ in two sub-networks, $M=M_{i n_{S}} \mid$ $M_{\text {out }_{S}}$ where $M_{i n_{S}}$ consists of all the nodes in $M$ belonging to $S$, while $M_{\text {out }}$ contains all the other nodes in the network. Then, by contextuality, it is sufficient to prove that

$$
M_{i n_{S}} \cong \operatorname{brd}\left(M_{i n_{S}}, l\right) \text {. }
$$

Indeed, this implies that

$$
n[P]_{l, r}^{\mu}\left|M_{\text {in }_{S}}\right| M_{\text {out }_{S}} \cong n[P]_{l, r}^{\mu}\left|\operatorname{brd}\left(M_{\text {in }_{S}}, l\right)\right| M_{\text {out }_{S}} .
$$

The following theorem proves the soundness of the above technique. Again the proof is reported in [5].

\section{Theorem 5.3: Given a network}

$$
M=n_{1}\left[P_{1}\right]_{l_{1}, r_{1}}^{\mu_{1}}|\ldots| n_{k}\left[P_{k}\right]_{l_{k}, r_{k}}^{\mu_{k}}
$$

and a location $l$, if $l$ is free of receiver-centered interference with respect to $M$ then $I_{r e c}(l, M)=0$.

\section{CONCLUSION}

Ad-hoc networks is a new area of mobile communication networks that has attracted significant attention due to its challenging problems.

The main goal of our work is to provide a formal model to reason about the problem of limiting the power consumption of communications. One of the most critical challenges in managing mobile ad-hoc networks is actually to find a good trade-off between network connectivity and power saving.
Even though not all the devices have the ability of adjusting their transmission power, modern technologies are quickly evolving, and there exist devices which are enabled to choose among two or more different power levels. For this reason many researches have proposed algorithms and protocols with the aim of providing a way to decide the best transmission power for node communications in a given network [2], [10], or to develop energy-aware routing protocols [3], [6].

In this paper we presented the E-BUM calculus which, due to its characteristics of modelling broadcast, multicast and unicast communications and also modelling the ability of a node to change its transmission power in accordance with the protocol it is executing, results to be a valid formal model for the analysis, evaluation and comparison of energyaware protocols and algorithms specifically developed for wireless ad-hoc networks. We also complemented the model by providing precise formal definitions of both sender- and receiver-centered interference.

\section{REFERENCES}

[1] M. Burkhart, P. von Rickenbach, R. Wattenhofer, and A. Zollinger. Does Topology Control Reduce Interference? In Proc. of the 5th Symposium on mobile Ad-hoc Networking and Computing, volume 623, pages 9-19. ACM, 2004

[2] T. Calamoneri, A. Clementi, A. Monti, G. Rossi, and R. Silvestri. Minimum-Energy Broadcast in Random-Grid Ad-Hoc Networks: Approximation and Distributed Algorithms. In MSWiM '08: Proc. of the 11th Int. Symposium on Modeling, Analysis and Simulation of Wireless and Mobile Systems, pages 354-361, New York, NY, USA, 2008. ACM.

[3] G. Ferrari, S. A. Malvassori, M. Bragalini, and O.K. Tonguz. Physical Layer-constrained Routing in Ad-hoc Wireless Networks: A Modified aodv Protocol with Power Control. In Int. Workshop on Wireless Ad-Hoc Networks 2005 (IWWAN'05), 2005

[4] M. Fusen, R. Wattenhofer, and A. Zollinger. Interference Arises at the Receiver. In Proc. of the Int. Conference on Wireless Networks, Communications, and Mobile Computing (WIRELESSCOM'05). IEEE press, 2005.

[5] L. Gallina and S. Rossi. A Process Calculus for Energy-Aware Multicast Communications of Mobile Ad-Hoc Networks. Research report, Department of Computer Science, University $\mathrm{Ca}$ ' Foscari of Venice, 2010. Available at http://www.dsi.unive.it/ srossi/E-BUM.pdf.

[6] J. Gomez and A. T. Campbell. Conserving Transmission Power in Wireless Ad Hoc Networks. In ICNP, pages 11-14, 2001.

[7] M. Merro. An Observational Theory for Mobile Ad Hoc Networks. Information and Computation, 207(2):194-208, 2009.

[8] F. Meyer auf de Heide, C. Schindelhauer, K. Volbert, and M. Grünewald. Energy, Congestion and Dilation in Radio Networks. In Proc. of the 14th Annual ACM Symposium on Parallel Algorithms and Architectures (SPAA'02), pages 230-237. ACM, 2002.

[9] R.Milner and D. Sangiorgi. Barbed Bisimulation. In Proc. of the Int. Colloquium on Automata, Languages and Programming (ICALP'92), volume 623 of LNCS, pages 685-695. Springer-Verlag, Berlin, 1992.

[10] M. Sanchez, P. Manzoni, and Z. J. Haas. Determination of Critical Transmission Range in Ad-Hoc Networks. In Proc. of the Multiaccess, Mobility and Teletraffic for Wireless Communications (MMT) Conference, 1999.

[11] A. Singh, C.R. Ramakrishnan, and S.A. Smolka. A Process Calculus for Mobile Ad Hoc Networks. In Proc. of the 10th Int. Conference on Coordination Models and Languages (COORDINATION'08), volume 5052 of LNCS, pages 296-314. Springer-Verlag, Berlin, 2008.

[12] P. von Rickenbach, S. Schmid, R. Wattenhofer, and A. Zollinger. A Robust Interference Model for Wireless Ad-Hoc Networks. In 5th Int. Workshop on Algorithms for Wireless, Mobile, Ad Hoc and Sensor Networks (WMAN). IEEE press, 2005. 